\title{
Distinct neural mechanisms for remembering when an event occurred
}

\author{
L. J. Jenkins ${ }^{1,{ }^{*}}$ and C. Ranganath ${ }^{2,3}$ \\ ${ }^{1}$ Department of Psychology, University of Wisconsin, Milwaukee P.O. Box 413, Milwaukee, WI \\ 53201 \\ 2Department of Psychology, University of California, Davis, 135 Young Hall, One Shields Ave., \\ Davis, CA 95616 \\ ${ }^{3}$ Center for Neuroscience, University of California, Davis, 1544 Newton Ct., Davis, CA 95618
}

\section{Abstract}

Events are often remembered as having occurred in a specific order, but almost nothing is known about how the brain encodes this temporal information. It is commonly assumed that temporal information is encoded via a single mechanism, based either on the temporal context in which the event occurred or inferred from the strength of the memory trace itself. By analyzing timedependent changes in activity patterns, we show that the distinctiveness of contextual representations in the hippocampus and anterior and medial prefrontal cortex was associated with accurate recency memory. In contrast, overall activation in the perirhinal and lateral prefrontal cortices predicted whether an object would be judged more recent, regardless of accuracy. These results demonstrate that temporal information is encoded through at least two complementary neural mechanisms.

\section{Keywords}

episodic memory; temporal order memory; recency discrimination; temporal context; functional MRI

Episodic memory, or memory for past events, is defined in part by the ability to remember when those events occurred (Tulving, 1972). Previous studies have shown that the medial temporal lobes (MTL; Fortin et al., 2002; Jenkins \& Ranganath, 2010; Tubridy \& Davachi, 2011) and prefrontal cortex (PFC; Jenkins \& Ranganath, 2010; Mangels, 1997; Milner et al., 1985; Shimamura et al., 1990) are critical to this ability, but almost nothing is known about the mechanisms by which these regions support memory for temporal information. One possibility, suggested by several computational models, is that events are bound at encoding to a representation of temporal context that changes gradually over time, and that it is this encoded context that conveys temporal information at retrieval (Bower, 1972; Howard \&

Kahana, 2002; Polyn et al., 2009). According to the logic of these models, an event would be

Corresponding Author: Lucas Jenkins, Department of Psychology, UW-Milwaukee, P.O.Box 413, Milwaukee, WI 53201, 414-316-1348, jenkinlj@uwm.edu. 
judged more recent than another event if its associated context were more similar to the current contextual state. An alternative possibility is that temporal information is inferred from the strength of the memories themselves, with stronger or more vivid memories perceived as more recent (Hinrichs, 1970; Hintzman, 2002, 2003, 2005).

In this functional magnetic resonance imaging (fMRI) study, we scanned participants as they encoded a series of object pictures and made recency judgments on subsequently presented picture pairs. We then attempted to identify sources of both context- and strength-based temporal information within the MTL and PFC using a novel combination of multivariate and univariate analyses. Because recency discrimination based on temporal context depends on the discriminability of the contextual information associated with each object, we expected that regions supporting this contextual representation would show multi-voxel activation patterns at encoding that were more dissimilar for objects that were recalled in the correct order (Manns et al., 2007). However, regions that represent item strength as the basis for subsequent recency discrimination should show greater univariate activation for the object that is judged to be more recent, regardless of whether that judgment is correct.

Seventeen participants were scanned as they performed eight sessions of the recency discrimination task. During the encoding period of each scanning run, participants were presented with 39 object photographs at a constant rate of one every $6 \mathrm{~s}$ and were instructed to make a semantic judgment for each object (Fig. 1). Following a $30 \mathrm{~s}$ filled delay, participants were then tested with 18 object pairs. Twelve of these pairs consisted of two objects that had appeared six objects apart during previous encoding period, and the remaining six pairs consisted of one object that had appeared earlier and one new object. Participants were instructed that if they recognized both objects, they were to indicate which of the two had appeared first and rate their confidence (High or Low). If they did not recognize both objects, they were to indicate that one of the objects was "New."

Performance on the behavioral task was relatively high, with $39 \%$ (SD 8\%) of recency pairs answered correctly with high confidence, $26 \%$ (SD 5\%) answered correctly with low confidence, and 33\% (SD 7\%) answered in the incorrect order. Only 3\% of recency pairs were rejected as new, compared to $97 \%$ of new object pairs (avg. $d^{\prime}=3.76$ ), indicating that incorrect responses on recency pairs were not due to a failure to recognize one or both objects.

To identify regions where the dissimilarity of encoded contextual representations predicted accurate recency discrimination at test, we first modeled the activity associated with each encoded object using separate regressors in a general linear model (GLM). We then conducted a searchlight analysis within our a priori MTL and PFC regions of interest contrasting the local multi-voxel pattern dissimilarity for pairs of objects recalled in the correct order with high confidence with pairs recalled in the incorrect order (Kriegeskorte et al., 2006). The results of this analysis are shown in Figure 2A. Three regions within the bilateral hippocampus (HPC) exhibited multi-voxel pattern dissimilarity that was significantly greater for correct than for incorrect object pairs: left (MNI: $-18,-34,4 ; t=$ $4.69, p=.03$ ) and right (MNI: $27,-39,3 ; t=4.38 ; p=.04$ ) posterior HPC, and right anterior 
HPC (MNI: $24,-12,-21 ; t=4.59, p=.05$ ). There were no significant clusters in which the opposite was true (i.e., greater pattern similarity for correct than for incorrect pairs).

Although the preceding analysis assumed a relationship between representational dissimilarity and recency accuracy that remained constant across the encoding period, it is also possible that successively encoded objects become more difficult to discriminate as the delay between study and test increases (Bjork \& Whitten, 1974; Yntema \& Trask, 1963). We therefore conducted a second searchlight analysis that regressed multi-voxel pattern dissimilarity for an object pair against both study-test delay and accuracy. As shown in Figure 2B, this analysis revealed a significant negative interaction in the medial (MNI: -9 , 35,$19 ; t=5.56$ ) and right anterior PFC (MNI: $24,50,37 ; t=5.21$ ), with pattern dissimilarity in these regions becoming less predictive of accuracy at longer delays. A post hoc comparison examining pattern dissimilarity separately at short and long delays confirmed that only at short delays $(<3 \mathrm{~min})$ did medial PFC pattern dissimilarity predict accurate recency judgments (Fig. 2C; $t=3.06, p=.004$, one-tailed).

To identify regions where the magnitude of activation at encoding predicted whether an object would be judged more recent, we estimated a new GLM with separate regressors modeling the first and second encoded objects for both high confidence correct and incorrect recency pairs. We then contrasted activity for all objects judged more recent (i.e., the second object in correct pairs, the first object in incorrect pairs) with activity for all objects judged less recent (i.e., the first object in correct pairs, the second object in incorrect pairs). As shown in Figure 3, this analysis revealed clusters that included the right perirhinal cortex (PRC) and HPC (MNI: $33-9-36 ; t=4.32, p=.04$ ) and the left lateral PFC (MNI: $-51,20$, $33 ; t=5.66, p=.05$ ) in which the strength of activation for one object relative to another predicted that that object would be judged more recent, regardless of whether that judgment were correct.

The results described here provide the first evidence, to our knowledge, that temporal information is encoded through multiple neural mechanisms. In the HPC and medial/anterior PFC, accurate recency discrimination was predicted by changes in the pattern of activation over time, consistent with a context-based source of temporal information. However, both correct and incorrect judgments of recency were predicted by the magnitude of activation in the PRC and lateral PFC, suggesting that these regions support temporal information based on memory strength. These results are consistent with behavioral studies suggesting that temporal order memory may be influenced by either the context (Block, 1972; Guenther \& Linton, 1975; Hupbach et al., 2007) or the strength (Hintzman, 2002, 2003, 2005) of encoded items or events.

Lesion studies in rodents (Fortin et al., 2002; Kesner et al., 2002), as well as human patient studies (Mayes et al., 2001; Spiers et al., 2001), have shown that damage to the HPC results in impaired performance on recency discrimination tasks despite relatively intact item recognition, and hippocampal activity during encoding has been shown in neuroimaging studies to predict memory for temporal order at retrieval (Dubrow \& Davachi, 2014; Jenkins \& Ranganath, 2010; Tubridy \& Davachi, 2011). These results are consistent with numerous studies implicating the HPC in episodic memory (Aggleton \& Brown, 1999; Eichenbaum et 
al., 2007) and indicate an important role in temporal order memory specifically, but they do not explain the mechanism by which the HPC supports memory for temporal information. Recent single-unit recording studies in rodents (MacDonald et al., 2011; Mankin et al., 2012; Manns et al., 2007; Pastalkova et al., 2008), monkeys (Naya \& Suzuki, 2011), and humans (Howard et al., 2012; Manning et al., 2011) have reported patterns of activity across ensembles of hippocampal neurons that change gradually over time, and on this basis it has been argued that the HPC may support an ongoing representation of temporal context (Eichenbaum, 2013). The results presented here bridge these earlier lines of research, demonstrating that changes in human hippocampal activation patterns over time predict memory for temporal order.

A different pattern of results was found in the PRC, where the strength of activation at encoding predicted whether an object would be judged more recent at test. Activity in the PRC has been shown in numerous studies to signal item recognition or item familiarity (Aggleton \& Brown, 1999; Diana et al., 2007; Eichenbaum et al., 2007; Xiang \& Brown, 1998), and our results suggest that this item strength signal may serve as a temporal cue as well. We note that the strength effect exhibited in the PRC also extended into the anterior HPC, although it did not overlap with the regions showing context-based effects. Judgments of memory strength have been shown in some cases to rely upon the vividness of the recollected detail (Brown et al., 1985; Hintzman, 2002), which raises the possibility that the effects in PRC and HPC may represent memory strength based on item and context information, respectively.

The finding that PFC activity also predicted memory for temporal order is consistent with lesions studies in rodents (Chiba et al., 1997; Cross et al., 2013) and monkeys (Fuster, 2008), as well as human patient studies (Milner et al., 1985; Shimamura et al., 1990), demonstrating that damage to the PFC results in deficits on recency discrimination tasks. It remains unclear why pattern dissimilarity in the medial and anterior PFC predicted accurate recency discrimination only at short delays. One possibility is that the PFC represents contextual information specific to current behavioral goals (Durstewitz et al., 2010; Hyman et al., 2012; Jenkins \& Ranganath, 2010; Preston \& Eichenbaum, 2013), which remain relatively constant during the typical fMRI experiment. These regions may therefore represent a more stable form of temporal context, conveying temporal information about objects encoded over longer time periods (Jenkins \& Ranganath, 2010) but insufficient to discriminate between successively encoded objects at longer delays.

\section{Detailed Methods}

\section{Participants}

Seventeen healthy young adults (8 male; mean age 24.6) were recruited from the UC Davis community and paid for their participation. All participants gave informed written consent before scanning. 


\section{Experimental Procedure}

Each functional run consisted of three parts: encoding, delay, and test. During the encoding period, participants were presented with 39 unique object photographs, appearing for $1.5 \mathrm{~s}$ each with a constant $4.5 \mathrm{~s}$ inter-trial interval (ITI). For each object, they were instructed to rate on a 4 point scale how likely it was that that object would be found "in the typical American home." This was followed by a $30 \mathrm{~s}$ filled delay period, during which participants viewed a series of simple arithmetic equations at a rate of one every $3 \mathrm{~s}$ and indicated whether each equation was true or false. They were then tested with 18 object pairs, appearing for $2 \mathrm{~s}$ each with a constant $4 \mathrm{~s}$ ITI. Twelve pairs consisted of two objects that had appeared six objects apart during the previous encoding period, and the remaining six pairs consisted of one object that had appeared earlier and one new object. Participants were instructed that if they recognized both objects they were to indicate which of the two had appeared first and rate their confidence (High or Low). If they did not recognize both objects, they were to indicate that one of the objects was "New." In order to minimize the influence of primacy and recency effects on responding, none of the tested objects were drawn from the first six or the last three objects of the encoding period. There were a total of eight functional runs, for a combined scanning time of approximately $50 \mathrm{~min}$.

\section{Image Acquisition and Pre-processing}

MRI data were acquired using a 3T Siemens Skyra scanner equipped with a 32-channel head coil. Functional images were acquired using a gradient echo planar imaging (EPI) sequence $(\mathrm{TR}=2000 \mathrm{~ms} ; \mathrm{TE}=25 \mathrm{~ms} ; \mathrm{FOV}=20.5 \times 21.12 \mathrm{~cm}$; image matrix $=64 \times 66$; flip angle $=$ 90; 34 sequential axial slices, $7 \%$ interslice gap; voxel size $=3.2 \times 3.2 \times 3.2 \mathrm{~mm}$ ). fMRI preprocessing and individual level statistical analyses were carried out using the SPM8 toolbox (http://www.fil.ion.ucl.ac.uk/spm/) and custom scripts. All functional images were initially realigned to the mean image using a six-parameter, rigid-body transform and sinc interpolated to correct for slice timing.

\section{Multivariate Analyses}

Multivariate analyses were conducted at the individual level using unsmoothed functional images resliced to a $3 \mathrm{~mm}$ isometric voxel size. The first step was to estimate a GLM that included separate regressors for each encoded object, along with regressors modeling delay and test period activity, motion artifacts, and session and baseline effects. The resulting object-specific beta maps were then subjected to a whole-brain searchlight analysis, in which a spherical searchlight with a two voxel radius was constructed around each voxel in the brain, and the pattern of beta values associated with each object was extracted from within that sphere. Each pattern was then normalized by subtracting the mean beta value and dividing by the standard deviation, and the difference between these normalized patterns was calculated for all tested object pairs using Euclidean distance as the multivariate distance metric. In order to correct for small variations in searchlight size, distance values were divided by the total number of voxels in each searchlight.

In the first reported analysis, the mean distance for pairs judged incorrectly at test was subtracted within each searchlight from the mean distance for pairs judged correctly with high confidence. In the second analysis, pattern distances for incorrect and high confidence 
correct pairs were regressed against both accuracy and the lag between encoding and test, and the coefficient of the interaction term was calculated within each searchlight. The individual distance maps produced by these analyses were then normalized to a common template (MNI), resliced into $3 \mathrm{~mm}$ isotropic voxels, and smoothed with a $3 \mathrm{~mm}$ full-width half-maximum (FWHM) Gaussian filter.

\section{Univariate Analysis}

Univariate analyses were conducted using normalized functional images, resliced to a $3 \mathrm{~mm}$ isotropic voxel size and smoothed with an $8 \mathrm{~mm}$ FWHM Gaussian filter. A new GLM was estimated for each participant that included separate regressors modeling the first and second encoded objects for both incorrect and high confidence correct pairs, with an additional regressor modeling the remaining encoded objects. All other design elements were identical to those of the previous analyses. Contrast images were calculated by subtracting the difference between the parameter estimates associated with the first and second objects in correct pairs from the difference between the first and second objects in incorrect pairs: $\left(\mathrm{Obj} 1_{\mathrm{Inc}}-\mathrm{Obj} 2_{\mathrm{Inc}}\right)-\left(\mathrm{Obj} 1_{\mathrm{Cor}}-\mathrm{Obj} 2_{\mathrm{Cor}}\right)$.

\section{Statistical Thresholding}

Group level statistical analyses were carried out using nonparametric randomization testing as implemented in FSL (http://fsl.fmrib.ox.ac.uk/fsl; 40). For each contrast, the null distribution of the maximum cluster mass was estimated by randomly flipping the sign of the individual statistical maps 10,000 times and thresholding at $t=3.0$. These distributions were used to identify clusters significant at a family-wise error rate of $p<.05$.

\section{Acknowledgments}

Grant sponsor: NIH; Grant number: 2ROIMH068721 and 5F31MH095445

\section{References}

Aggleton JP, Brown MW. 1999; Episodic memory, amnesia, and the hippocampal-anterior thalamic axis. Behavioral Brain Science. 22:425-489.

Bjork RA, Whitten WB. 1974; Recency-sensitive retrieval processes in long-term free recall. Cognitive Psychology. 6:173-189.

Block RA. 1972; Temporal judgments and contextual change. Journal of Experimental Psychology: Learning, Memory, and Cognition. 8:530-544.

Bower, GH. Stimulus-sampling theory of encoding variability. In: Melton, AW, Martin, E, editors. Coding Processes in Human Memory. John Wiley and Sons; 1972. 85-121.

Brown NR, Rips LJ, Shevell SK. 1985; The subjective dates of natural events in very-long-term memory. Cognitive Psychology. 17:139-177.

Chiba AA, Kesner RP, Gibson CJ. 1997; Memory for temporal order of new and familiar spatial location sequences: role of the medial prefrontal cortex. Learning and Memory. 4:311-317. [PubMed: 10706368]

Cross L, Brown MW, Aggleton JP, Warburton EC. 2013; The medial dorsal thalamic nucleus and the medial prefrontal cortex of the rat function together to support associative recognition and recency but not item recognition. Learning and Memory. 20:41-50.

Diana RA, Yonelinas AP, Ranganath C. 2007; Imaging recollection and familiarity in the medial temporal lobe: a three-component model. Trends in Cognitive Sciences. 11:379-386. [PubMed: 17707683] 
Dubrow S, Davachi L. 2014; Temporal memory is shaped by encoding stability and intervening item reactivation. Journal of Neuroscience. 34:13998-14005. [PubMed: 25319696]

Durstewitz D, Vittoz NM, Floresco SB, Seamans JK. 2010; Abrupt transitions between prefrontal neural ensemble states accompany behavioral transitions during rule learning. Neuron. 66:438448. [PubMed: 20471356]

Eichenbaum H. 2013; Memory on time. Trends in Cognitive Science. 17:81-88.

Eichenbaum H, Yonelinas AP, Ranganath C. 2007; The medial temporal lobe and recognition memory. Annual Review of Neuroscience. 30:123-152.

Fortin NJ, Agster KL, Eichenbaum HB. 2002; Critical role of the hippocampus in memory for sequences of events. Nature Neuroscience. 5:458-462. [PubMed: 11976705]

Fuster, JM. The Prefrontal Cortex. 4. Academic Press; London: 2008.

Guenther RK, Linton M. 1975; Mechanisms of temporal coding. Journal of Experimental Psychology: Human Learning and Memory. 97:220-229.

Hinrichs JV. 1970; A two-process memory-strength theory for judgment of recency. Psychological Review. 77:223-233.

Hintzman DL. 2002; Context matching and judgments of recency. Psychonomic Bulletin and Review. 9:368-374. [PubMed: 12120802]

Hintzman DL. 2003; Judgments of recency and their relation to recognition memory. Memory and Cognition. 31:26-34. [PubMed: 12699140]

Hintzman DL. 2005; Memory strength and recency judgments. Psychonomic Bulletin and Review. 12:858-864. [PubMed: 16524002]

Howard MW, Kahana MJ. 2002; A distributed representation of temporal context. Journal of Mathematical Psychology. 46:269-299.

Howard MW, Viskontas IV, Shankar KH, Fried I. 2012; Ensembles of human MTL neurons "jump back in time" in response to a repeated stimulus. Hippocampus. 22:1833-1847. [PubMed: 22488671]

Hupbach A, Gomez R, Hardt O, Nadel L. 2007; Reconsolidation of episodic memories: A subtle reminder triggers integration of new information. Learning \& Memory. 14:47-53. [PubMed: 17202429]

Hyman JM, Ma L, Balaguer E, Durstewitz D, Seamans JK. 2012; Contextual encoding by ensembles of medial prefrontal cortex neurons. Proceedings of the National Academy of Sciences. 109:50865091.

Jenkins LJ, Ranganath C. 2010; Prefrontal and medial temporal lobe activity at encoding predicts temporal context memory. Journal of Neuroscience. 30:15558-15565. [PubMed: 21084610]

Kesner RP, Gilbert PE, Barua LA. 2002; The role of the hippocampus in memory for the temporal order of a sequence of odors. Behavioral Neuroscience. 116:286-290. [PubMed: 11996313]

Kriegeskorte N, Goebel R, Bandettini P. 2006; Information-based functional brain mapping. Proceedings of the National Academy of Sciences. 103:3863-3868.

MacDonald CJ, Lepage KQ, Eden UT, Eichenbaum H. 2011; Hippocampal "time cells" bridge the gap in memory for discontiguous events. Neuron. 71:737-749. [PubMed: 21867888]

Mangels JA. 1997; Strategic processing and memory for temporal order in patients with frontal lobe lesions. Neuropsychology. 11:207-221. [PubMed: 9110328]

Mankin EA, Sparks FT, Slayyeh B, Sutherland RJ, Leutgeb S, Leutgeb J. 2012; Neuronal code for extended time in the hippocampus. Proceedings of the National Academy of Sciences. 109:1946219467.

Manning JR, Polyn SM, Baltuch GH, Litt B, Kahana MJ. 2011; Oscillatory patterns in temporal lobe reveal context reinstatement during memory search. Proceedings of the National Academy of Sciences. 108:12893-12897.

Manns JR, Howard MW, Eichenbaum H. 2007; Gradual changes in hippocampal activity support remembering the order of events. Neuron. 56:530-540. [PubMed: 17988635]

Mayes AR, et al. 2001; Memory for single items, word pairs, and temporal order of different kinds in a patient with selective hippocampal lesions. Cognitive Neuropsychology. 18:97-123. [PubMed: 20945208] 
Milner B, Petrides M, Smith ML. 1985; Frontal lobes and the temporal organization of memory. Human Neurobiology. 4:137-42. [PubMed: 4066424]

Naya Y, Suzuki WA. 2011; Integrating what and when across the primate medial temporal lobe. Science. 333:773-776. [PubMed: 21817056]

Pastalkova E, Itskov V, Amarasingham A, Buzsaki G. 2008; Internally generated cell assembly sequences in the rat hippocampus. Science. 324:1322-1327.

Polyn SM, Norman KA, Kahana MJ. 2009; A context maintenance and retrieval model of organizational processes in free recall. Psychological Review. 116:129-156. [PubMed: 19159151]

Preston AR, Eichenbaum H. 2013; Interplay of hippocampus and prefrontal cortex in memory. Current Biology. 23:R764-R773. [PubMed: 24028960]

Shimamura AP, Janowsky JS, Squire LR. 1990; Memory for the temporal order of events in patients with frontal lobe lesions and amnesic patients. Neuropsychologia. 28:803-813. [PubMed: 2247207]

Spiers HJ, Burgess N, Hartley T, Vargha-Khadem F, O’Keefe J. 2001; Bilateral hippocampal pathology impairs topographical and episodic memory but not visual pattern matching. Hippocampus. 11:715-725. [PubMed: 11811666]

Tubridy S, Davachi L. 2011; Medial temporal lobe contributions to episodic sequence encoding. Cerebral Cortex. 21:272-280. [PubMed: 20494967]

Tulving, E. Episodic and semantic memory. In: Tulving, E, Donaldson, W, editors. Organization and memory. Academic Press; New York: 1972.

Xiang JZ, Brown MW. 1998; Differential neuronal encoding of novelty, familiarity and recency in regions of the anterior temporal lobe. Neuropharmacology. 37:657-676. [PubMed: 9705004]

Yntema DB, Trask FP. 1963; Recall as a search process. Journal of Verbal Learning and Verbal Behavior. 2:65-74. 


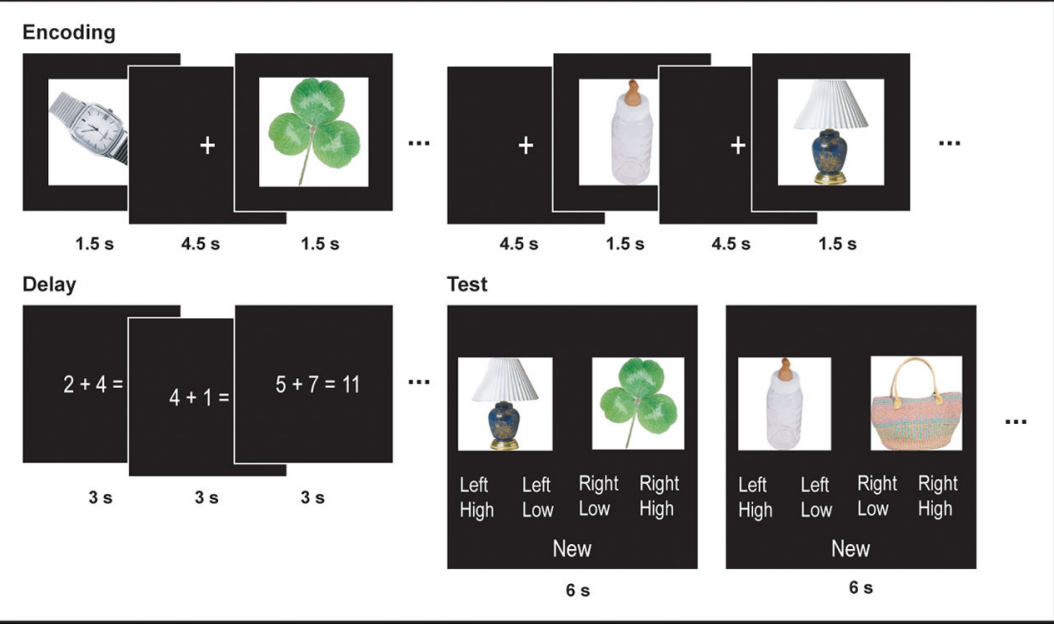

Figure 1.

Diagram of experimental task. Thirty-nine object photographs were presented at $6 \mathrm{~s}$ intervals in the context of a semantic judgment task. Following a $30 \mathrm{~s}$ filled delay, participants were tested with 18 object pairs. Twelve pairs consisted of two objects that had appeared six object apart in the previous list, and participants were to select the object that had appeared earlier. Six pairs consisted of one old and one new object, and participants were to select "New". 


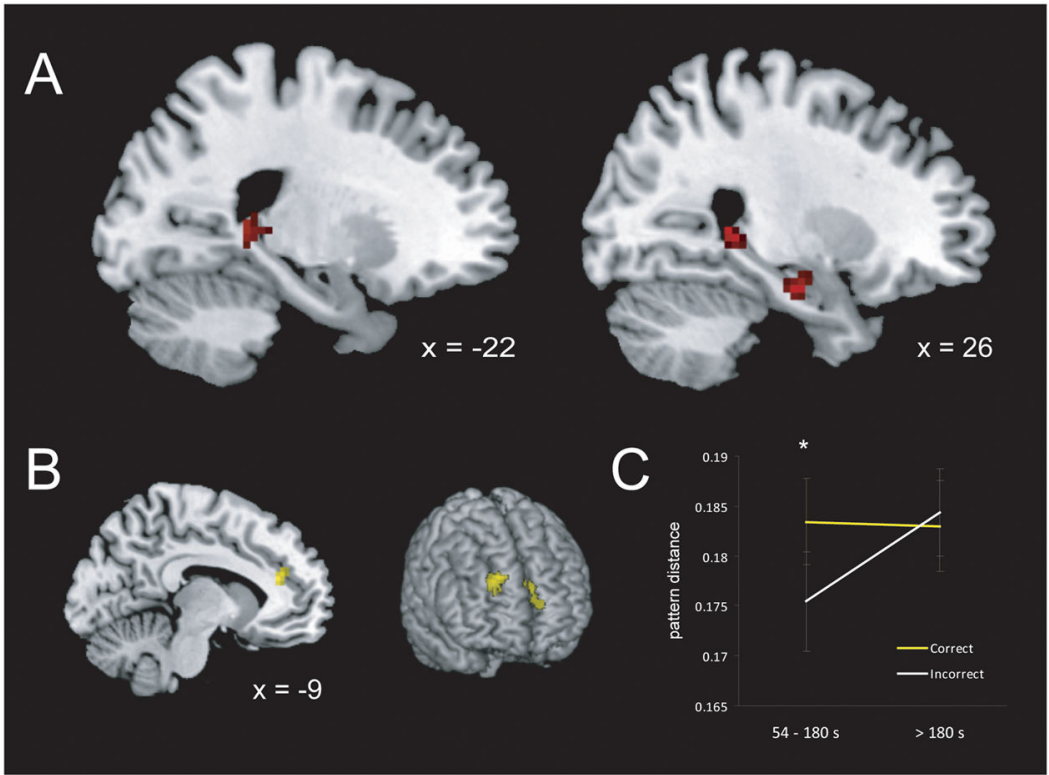

Figure 2.

(A) Pattern dissimilarity in the bilateral posterior HPC and right anterior HPC at encoding predicted accurate order judgments at test. (B) Pattern dissimilarity in the medial and anterior PFC interacted with study/test lag to predict accuracy at test. (C) Pattern dissimilarity within the medial PFC predicted accuracy only at shorter lags $\left(<3 \mathrm{~min} ; t_{1,16}=\right.$ $3.06, p=.004$, one-tailed). Error bars denote $+/-1$ SEM. 


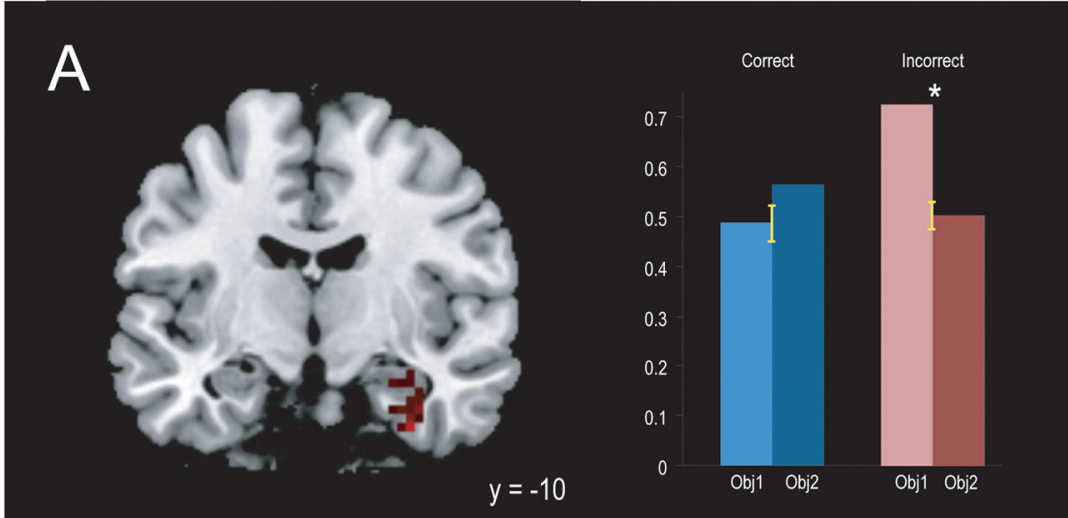

\section{B}

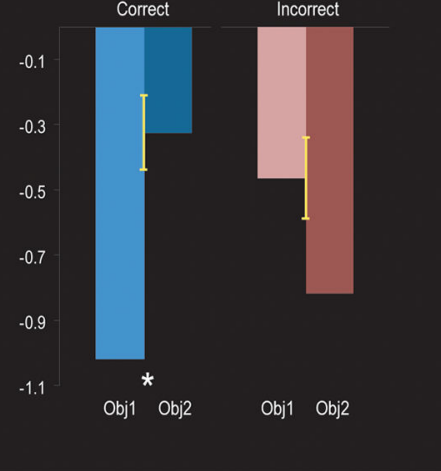

Figure 3.

Activation in the right PRC and anterior HPC (A) and left lateral PFC (B) exhibited a significant interaction between Object (Object 1, Object 2) and Accuracy (Correct, Incorrect). (A) In the PRC and HPC, activation was significantly greater for the first object in incorrect pairs $\left(t_{(16)}=4.02, p=<.001\right)$, with no significant difference between the first and second objects in correct pairs $\left(t_{(16)}=1.02, p=.16\right)$. (B) In the lateral PFC, activation was significantly greater for the second object in correct pairs $\left(t_{(16)}=3.08, p=.004\right)$, and marginally greater for the first object in incorrect pairs $\left(t_{(16)}=1.42, p=.08\right)$. Error bars denote +/- 1 SEM. 